\title{
Preparation, characterization and in vivo evaluation of bergenin-phospholipid complex
}

\author{
Xuan QIN ${ }^{1}$, Yang YANG ${ }^{1}$, Ting-ting FAN ${ }^{1}$, Tao GONG ${ }^{1}$, Xiao-ning ZHANG ${ }^{2}$, Yuan HUANG ${ }^{1, *}$ \\ ${ }^{1}$ Key Laboratory of Drug Targeting and Drug Delivery System, Ministry of Education, West China School of Pharmacy, Sichuan Univer- \\ sity, Chengdu 610041, China; ${ }^{2}$ School of Medicine, Tsinghua University, Beijing 100084, China
}

Aim: To prepare a bergenin-phospholipid complex (BPC) to increase oral bioavailability of the drug.

Methods: In order to obtain the acceptable BPC, a spherical symmetric design-response surface methodology was used for process optimization. The influence of reaction medium, temperature, drug concentration and drug-to-phospholipid ratio on the combination percentage and content of bergenin in BPC were evaluated. BPC was then characterized by thin-layer chromatography (TLC), high-performance liquid chromatography (HPLC), ultra-violet (UV) spectroscopy, fourier transform infrared spectroscopy (FT-IR), differential scanning calorimetry (DSC) and X-ray powder diffraction. The physicochemical properties such as microscopic shape, particle size, zetapotential, solubility, crystalline form, and hygroscopicity were tested. The pharmacokinetic characteristics and bioavailability of BPC were investigated after oral administration in rats in comparison to bergenin and the physical mixture (bergenin and phospholipids). Results: BPC was successfully prepared under the optimum conditions [temperature $=60{ }^{\circ} \mathrm{C}$, drug concentration $=80 \mathrm{~g} / \mathrm{L}$ and drug-tophospholipids ratio $=0.9(\mathrm{w} / \mathrm{w})]$. The combination percentage was $100.00 \% \pm 0.20 \%$, and the content of bergenin in the complex was $45.98 \% \pm 1.12 \%$. Scanning electron microscopy and transmission electron microscopy of BPC showed spherical particles. The average particle size was $169.2 \pm 20.11 \mathrm{~nm}$ and the zeta-potential was $-21.6 \pm 2.4 \mathrm{mV}$. The solubility of BPC in water and in $n$-octanol was effectively enhanced. The $C_{\max }$ and $\mathrm{AUC}_{0 \rightarrow \infty}$ of BPC were increased, and the relative bioavailability was significantly increased to $439 \%$ of bergenin.

Conclusion: The BPC is a valuable delivery system to enhance the oral absorption of bergenin.

Keywords: bergenin; phospholipid complex; spherical symmetric design-response surface methodology; physicochemical properties; bioavailability

Acta Pharmacologica Sinica (2010) 31: 127-136; doi: 10.1038/aps.2009.171; published online 7 December 2009

\section{Introduction}

Bergenin (3,4,8,10-tetrahydroxy-2-hydroxymethyl-9methoxy-2,3,4,4a-tetrahydropyrano[3,2-c]isochromen-6one) is a major component of traditional Chinese medicine Bergenia crassiflora ${ }^{[1,2]}$. Bergenin has been widely used for the treatment of chronic bronchitis and has other effects such as antitussive, hepatoprotective, anti-inflammatory, neuroprotective and weak anti-HIV activities ${ }^{[3-6]}$. Although bergenin has been used for more than 30 years, its poor oral bioavailability is still an obstacle to its further application ${ }^{[7]}$. The Biopharmaceutics Classification System (BCS) classifies drugs into four categories depending on their solubility and permeability characteristics $^{[8]}$. According to this scheme, as a class IV com-

\footnotetext{
* To whom correspondence should be addressed.

E-mail huangyuan0@yahoo.com.cn

Received 2009-07-20 Accepted 2009-10-29
}

pound, bergenin has neither sufficient solubility nor permeability for complete absorption ${ }^{[9]}$. Furthermore, conventional tablet is the formulation of bergenin on the market, but a high dose $(375 \mathrm{mg} / \mathrm{d})$ has to be used in order to exert therapeutic effects. Many efforts have been made to enhance its bioavailability, such as structure modification, prodrugging and new dosage forms such as dripping pills, soft capsules and $\beta$-cyclodextrin inclusion complexes ${ }^{[10-14]}$. However, no data has been published in relation to the improved bioavailability after oral administration.

In this study, a phospholipid complex was adopted to increase both the water solubility and liposolubility of bergenin to enhance its oral bioavailability. The preparation of the bergenin-phospholipid complex (BPC) was optimized by a spherical symmetric design-response surface methodology. Furthermore, the physicochemical properties and oral bioavailability of BPC was explored in comparison to bergenin 
and the physical mixture (bergenin and phospholipids).

\section{Materials and methods Materials}

Bergenin was kindly supplied by Sichuan Dihon Medical Development Co, Ltd (Chengdu, China). Phospholipid was purchased from Shanghai Toshisun Enterprise Co, Ltd (Shanghai, China), and the phosphatidyl content was approximately $80 \%(w / w)$. Methanol and water were of HPLC grade. All the other reagents and solvents were of the highest purity commercially available.

\section{Chromatography}

A sensitive HPLC assay was developed to analyze the content of bergenin. The HPLC instrument employed was an Alltech $^{\mathrm{TM}}$ (manufactured by Alltech Technologies, USA) LC system with a model 426 pump and model UVIS-201 absorbance detector. The output signal was monitored and processed using the AllChrom ${ }^{\mathrm{TM}}$ Plus Chromatograph Data System (designed by Multilink Services Co Ltd). The columns were Dikma Diamonsil ${ }^{\circledR} C_{18}(150 \times 4.6 \mathrm{~mm}, 5 \mu \mathrm{m})$ and Dikma EasyGuard $6101 \mathrm{C}_{18}$ kit guard column. The mobile phase was a mixture of methanol-water $(20: 80, v / v, \mathrm{pH} 2.50)$ at a flow rate of $1 \mathrm{~mL} / \mathrm{min}$. The wavelength was set at $275 \mathrm{~nm}$. All analyses were performed at $30^{\circ} \mathrm{C}$.

\section{Preparation of BPC}

A weighed amount of bergenin and phospholipids were charged in a round bottom flask and dissolved in anhydrous ethanol. The mixture was refluxed at a controlled temperature for about $2 \mathrm{~h}$. The resultant clear solution was evaporated and dried under vacuum $\left(40^{\circ} \mathrm{C}\right)$. The residues were then gathered and placed in desiccators.

Preliminary investigations of the process parameters revealed that reaction temperature $\left(X_{1}\right)$, drug concentration $\left(X_{2}\right)$ and the drug to phospholipid ratio $\left(X_{3}\right)$ highly influenced both the combination percentage $\left(Y_{1}\right)$ and content of bergenin $\left(Y_{2}\right)$. A statistical model incorporating interactive and polynomial terms was used to evaluate the response employing the equation:

$Y=b_{0}+b_{1} X_{1}+b_{2} X_{2}+b_{3} X_{3}+b_{4} X_{1}^{2}+b_{5} X_{2}^{2}+b_{6} X_{3}^{2}+b_{7} X_{1} X_{2}+b_{8} X_{1} X_{3}+b_{9} X_{2} X_{3}$

where $Y$ was the dependent variable, $b_{0}$ was the intercept representing the arithmetic average of the 15 runs, and $b_{1}$ to $b_{9}$ were the estimated coefficients for the factors $\left(X_{i}, i=1,2\right.$, 3). $X_{1}, X_{2}$, and $X_{3}$ were the coded levels of the independent variable(s). The interaction terms $X_{1} X_{2}, X_{1} X_{3}$, and $X_{2} X_{3}$ showed how the response changed when two factors were changed simultaneously. The polynomial terms $\left(X_{i}^{2}\right)$ were included to investigate nonlinearity. The level values of three factors and the composition of central composite design batches 1 to 15 are shown in Tables 1 and 2 .

The combination percentage of bergenin was calculated using the following equation: Combination percentage $(\%)=(A-B) / A * 100 \%$, where $A$ was the weighed amount of
Table 1. Independent variables and their correspondent values for optimization of BPC preparation using the spherical symmetric designresponse surface methodology.

\begin{tabular}{lccccc}
\hline Variables & \multicolumn{7}{c}{ Levels } \\
& -1.732 & -1 & 0 & 1 & 1.732 \\
\hline$X_{1}$ & 30 & 38.45 & 50 & 61.55 & 70 \\
$X_{2}$ & 20 & 36.91 & 60 & 83.09 & 100 \\
$X_{3}$ & 0.4 & 0.57 & 0.8 & 1.03 & 1.2 \\
\hline
\end{tabular}

Table 2. Response values of different variables for the optimization of BPC preparation using the spherical symmetric design-response surface methodology.

\begin{tabular}{cccccc}
\hline $\begin{array}{c}\text { Formula- } \\
\text { tion }\end{array}$ & $X_{1}$ & $X_{2}$ & $X_{3}$ & $Y_{1}$ & $Y_{2}$ \\
\hline 1 & -1 & -1 & -1 & 100.00 & 34.70 \\
2 & -1 & -1 & 1 & 98.82 & 47.02 \\
3 & -1 & 1 & -1 & 67.19 & 26.76 \\
4 & -1 & 1 & 1 & 76.19 & 43.06 \\
5 & 1 & -1 & -1 & 97.82 & 37.22 \\
6 & 1 & -1 & 1 & 90.25 & 46.13 \\
7 & 1 & 1 & -1 & 98.44 & 35.91 \\
8 & 1 & 1 & 1 & 96.85 & 47.07 \\
9 & -1.732 & 0 & 0 & 65.27 & 32.97 \\
10 & 1.732 & 0 & 0 & 99.12 & 43.50 \\
11 & 0 & -1.732 & 0 & 100.00 & 45.78 \\
12 & 0 & 1.732 & 0 & 90.11 & 45.36 \\
13 & 0 & 0 & -1.732 & 100.00 & 26.94 \\
14 & 0 & 0 & 1.732 & 90.74 & 49.85 \\
15 & 0 & 0 & 0 & 100.00 & 41.41 \\
\hline
\end{tabular}

bergenin for preparing the complex and B was the content not forming a complex. The content of bergenin in the complex was estimated by HPLC. It was calculated as: content $(w /$ $w, \%)=C / D^{*} 100 \%$, where $C$ was the amount of bergenin that formed a complex and D was the weight of the BPC.

\section{Thin-layer chromatography (TLC)}

Sample solutions were prepared by dissolving bergenin, phospholipids, the physical mixture and BPC in methanol. TLC plates were precoated with silica gel at a $0.15-0.2 \mathrm{~mm}$ thickness. The plates were saturated with a solvent system of acetic ether:methanol $(5: 1, v / v)$, developed to a distance of about 4.5 $\mathrm{cm}$ and removed from the chamber to dry in air. Phospholipids were visualized by ninhydrin, and the spot of bergenin was observed with a ZF-I ultraviolet analysis instrument (Shanghai Gucun Optic Instrument Factory, China).

\section{Ultraviolet (UV) spectra, HPLC, and Fourier Transform Infrared spectra (FT-IR)}

Tested samples were dissolved in water and then scanned with a UV spectrometer (Varian Cary 100, America) over the 
wavenumber range of 200-600 nm. For HPLC, a $10 \mu \mathrm{L}$ aliquot sample solution was injected into the HPLC. The FT-IR spectra were recorded on an FT-IR spectrometer (VECTOR 22, Bruker, Germany) after the samples were compressed into a $\mathrm{KBr}$ pellet.

\section{Differential scanning calorimetry (DSC)}

The samples were sealed in an aluminum crimp cell and heated at $5{ }^{\circ} \mathrm{C} / \mathrm{min}$ from 0 to $300{ }^{\circ} \mathrm{C}$ in a nitrogen atmosphere at a flow rate of $50 \mathrm{~mL} / \mathrm{min}$. The peak transition onset temperature of bergenin, phospholipids, BPC and the physical mixture were determined and compared with the help of a differential scanning calorimeter (EXSTAR6000 DSC, Japan).

\section{X-ray powder diffraction}

The X-ray diffraction was recorded on an X-ray diffractometer (PHILIPS X'Pert Pro MPD DY1291, Japan). Samples of certain weight were added into the slide for packing prior to X-ray scanning. Spectra of graphs were plotted from $3.00^{\circ}$ to $65.00^{\circ}$ of $2 \theta$ with a step width of $0.03^{\circ}$ and step time of $0.2 \mathrm{~s}$ at room temperature.

\section{Morphological characteristics}

The samples were coated with platinum in a sputter coater, and their surface morphology was viewed and photographed with a scanning electron microscope (SEM). A transmission electron microscope (TEM, H-600IV, Hitachi, Japan) was used for microscopic characterization of the complex. The complex was suspended in distilled water, and a drop was placed on a carbon-coated copper grid. The films on the grid were negatively stained by immediately adding a drop of $2 \%(w / w)$ ammonium molybdate in $2 \%(w / v)$ ammonium acetate buffer ( $\mathrm{pH}$ 6.8), removing the excess staining solution with a filter paper and air-drying.

\section{Particle size and zeta-potential}

The particle size and zeta-potential of BPC were determined at $25^{\circ} \mathrm{C}$ using photon correlation spectroscopy (Malvern ZetaSize Nano ZS90, UK). A proper dilution of the suspension was prepared with double-distilled water before each analysis.

\section{Solubility studies}

Solubility determination was carried out by adding an excess of sample to $5 \mathrm{~mL}$ of water or $n$-octanol in sealed glass containers at $25^{\circ} \mathrm{C}$. Each experiment was performed in triplicate. The liquids were agitated for $24 \mathrm{~h}$ and then centrifuged to remove solid substances $(12000 \times g, 5 \mathrm{~min})$. The supernatant was filtered through a $0.45 \mu \mathrm{m}$ membrane. Then, $1 \mathrm{~mL}$ of filtrate was mixed with $9 \mathrm{~mL}$ of solvent, and a $10 \mu \mathrm{L}$ aliquot of the resulting solution was injected into an HPLC.

\section{Hygroscopicity}

Solid samples were stored under controlled temperature and humidity conditions to investigate the ability of bergenin and BPC to take up water from the environment. The relative humidity $(\mathrm{RH})$ at $25{ }^{\circ} \mathrm{C}$ was prepared using saturated solutions with known RH values $(22.45 \%, 42.76 \%, 57.7 \%$, $75.28 \%, 84.26 \%, 92.50 \%$, and $98.00 \%$ ) in desiccators ${ }^{[15]}$. Certain amounts of samples were weighed and put into open clear glass bottles that were exposed to the desired $\mathrm{RH}$. The gain in weight of the samples was determined up to saturation humidity. At each investigated $\mathrm{RH}$, samples were prepared in triplicate.

\section{Pharmacokinetic study in rats \\ Experiment design}

Male Wistar rats $(250 \pm 20 \mathrm{~g})$ were obtained from the Laboratory Animal Center of Sichuan University. All experiments were approved by the Institutional Animal Care and Use Committee of Sichuan University. Animals were divided randomly into three groups $(n=5)$. Each group of rats was given a single oral dose of free bergenin, the physical mixture or BPC (equivalent to $300 \mathrm{mg}$ of bergenin). Blood samples were obtained at appropriate time points; $0.2 \mathrm{~mL}$ of plasma was separated by centrifugation $(12000 \times g)$ for $5 \mathrm{~min}$ and then stored at $-20^{\circ} \mathrm{C}$ until analysis.

\section{Plasma sample preparation}

The preparation method of the plasma samples was developed in our laboratory to quantify the bergenin concentration in biosamples ${ }^{[16]}$. When the plasma sample was thawed, 20 $\mu \mathrm{L}$ of the IS solution was added and agitated for $20 \mathrm{~s}$. Then $600 \mu \mathrm{L}$ of ethyl acetate was added and shaken for $2 \mathrm{~min}$, and sonic oscillation was performed to obtain complete extraction. The extracts were then centrifuged at $3000 \times g$ to achieve better phase separation. This procedure was repeated three times, and all of the supernatant was mixed and dried under vacuum. The residues were reconstituted with $100 \mu \mathrm{L}$ of mobile phase and then centrifuged at $12000 \times g$ for $2 \mathrm{~min}$. A 20 $\mu \mathrm{L}$ aliquot of the supernatant was then injected into the HPLC system.

\section{Pharmacokinetic parameters}

The pharmacokinetic parameters for bergenin in plasma were estimated by appropriate compartmental methods. All parameters were determined from the sample collection times and the assayed concentrations at these time points. Plasma concentrations were plotted against time, and the pharmacokinetic calculations were performed using the standard software program DAS 2.0 (Drug and Statistics, Anhui, China). The following parameters were estimated: absorption half-life $\left(t_{1 / 2 \alpha}\right)$, elimination half-life $\left(t_{1 / 2 \beta}\right)$, maximal plasma concentration $\left(C_{\max }\right)$, area under the plasma concentration time curve (AUC), and mean residence time (MRT). Free bergenin was taken as the control group to calculate the relative BA values.

\section{Results}

\section{Preparation of BPC}

The experimental results concerning the tested variables on the combination or content of bergenin in the complex are shown in Table 2. A mathematical relationship between factors and parameters was generated by response surface regres- 

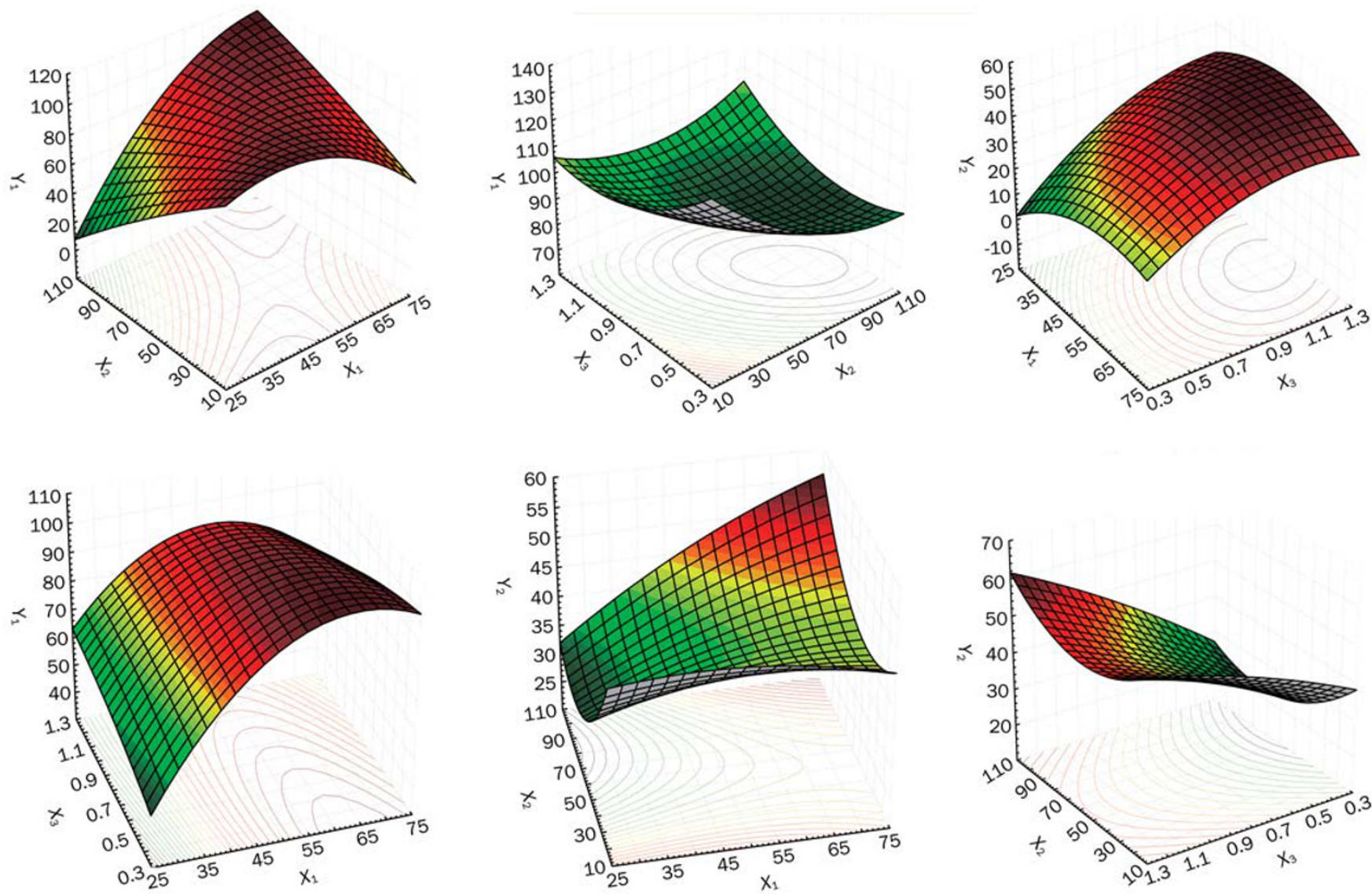

Figure 1. Response surface plot showing the influence of reaction temperature $\left(X_{1}\right)$, drug concentration $\left(X_{2}\right)$, drug to phospholipids ratio $\left(X_{3}\right)$ on combination percentage $\left(Y_{1}\right)$ and content of bergenin $\left(Y_{2}\right)$.

sion analysis in the software STATISTICA 6.0. The threedimensional response surface plots for the most statistically significant variables on the evaluated responses are shown in Figure 1. The equations represented the quantitative effect of process variables $\left(X_{1}, X_{2}\right.$, and $\left.X_{3}\right)$, and their interactions on their responses $Y_{1}$ and $Y_{2}$ are listed as follows:

\section{$Y_{1}=17.8292+3.9683 X_{1}-1.5953 X_{2}+59.0044 X_{3}-0.0448 X_{1}^{2}-0.0032 X_{2}^{2}$ $-29.5646 X_{3}^{2}+0.0294 X_{1} X_{2}-0.7960 X_{1} X_{3}+0.3788 X_{2} X_{3}$}

\section{$Y_{2}=-10.7459+1.1372 X_{1}-0.6964 X_{2}+74.4763 X_{3}-0.0094 X_{1}^{2}+0.0022 X_{2}^{2}$ $-22.4228 X_{3}^{2}+0.0054 X_{1} X_{2}-0.4008 X_{1} X_{3}+0.1462 X_{2} X_{3}$}

The polynomial models for $Y_{1}$ and $Y_{2}$ were found to be significant, with $F$ values of 482.45 and 760.97 , respectively $(P<0.05)$. The values of correlation coefficients $(R)$ were found to be 0.9670 and 0.9892 , respectively, indicating a good fit to the quadratic model.

The response surface and contour plots (Figure 1) clearly indicated that $X_{1}, X_{2}$, and $X_{3}$ strongly influenced the combination percentage $\left(Y_{1}\right)$ and content of bergenin $\left(Y_{2}\right)$. The combination (\%) for the 15 batches showed a wide variation of $65.27 \%$ to $100.00 \%$, and the content was in the range of $26.94 \%$ to $49.85 \%$ (Table 2). It was found that an increase in reaction temperature led to an increase of combination percentage and drug content. The drug-to-phospholipids ratio was an important factor affecting the drug content.

In order to evaluate the optimization capability of the models generated according to the results of the spherical symmetric design-response surface methodology, the BPC was prepared using the optimal process variable settings, where $X_{1}, X_{2}$, and $X_{3}$ were equal to $60^{\circ} \mathrm{C}, 80 \mathrm{~g} / \mathrm{L}$ and 0.9 , respectively (Table 3). The combination percentage was $100.00 \%$, and the content of bergenin in the complex was $45.98 \% \pm 1.12 \%$. The results illustrated a good relationship between the experimental and predicted values, which confirmed the practicability and validity of the model. The predicted error for all the response variables was below $3 \%$, indicating that the optimization technique was appropriate for optimizing BPC.

Table 3. Comparison of the observed and predicted values in BPC prepared under predicted optimum conditions.

\begin{tabular}{lccc}
\hline Response variable & Predicted value & Observed value & Bias (\%) \\
\hline Combination (\%) & 100.00 & $100.00 \pm 0.20$ & 0 \\
Content (\%) & 47.37 & $45.98 \pm 1.12$ & -2.93 \\
\hline
\end{tabular}




\section{Thin-layer chromatography (TLC)}

The chromatogram of BPC was the same as the physical mixture. Two spots were observed (Figure 2): one was similar to the phospholipids (on the starting line), and the other had the same $R_{\mathrm{f}}$ as bergenin $\left(R_{\mathrm{f}}=0.58\right)$. No new spot was found.

\section{UV, HPLC, and FT-IR spectrum}

The UV spectra are shown in Figure 3. The phospholipids showed only end absorptions close to $200 \mathrm{~nm}$. The absorption curves of bergenin, the physical mixture and BPC were nearly the same. Two characteristic absorption bands of bergenin were observed at 220 and $275 \mathrm{~nm}$, which was in accordance with the Pharmacopoeia of PRC ${ }^{[17]}$. The retention time of BPC was the same as bergenin, and no new peak was present in the HPLC chromatogram (Figure 4). As shown in Figure 5,

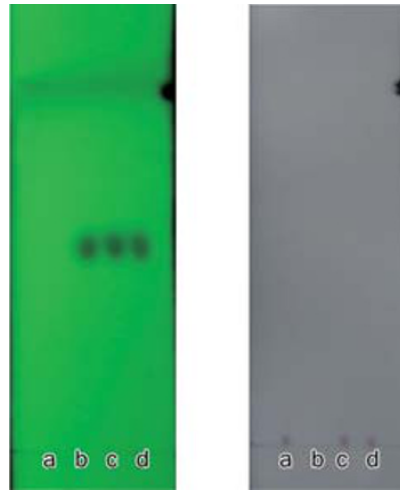

Figure 2. TLC of phospholipids (a), bergenin (b), physical mixture (c), and $\operatorname{BPC}(d)$.
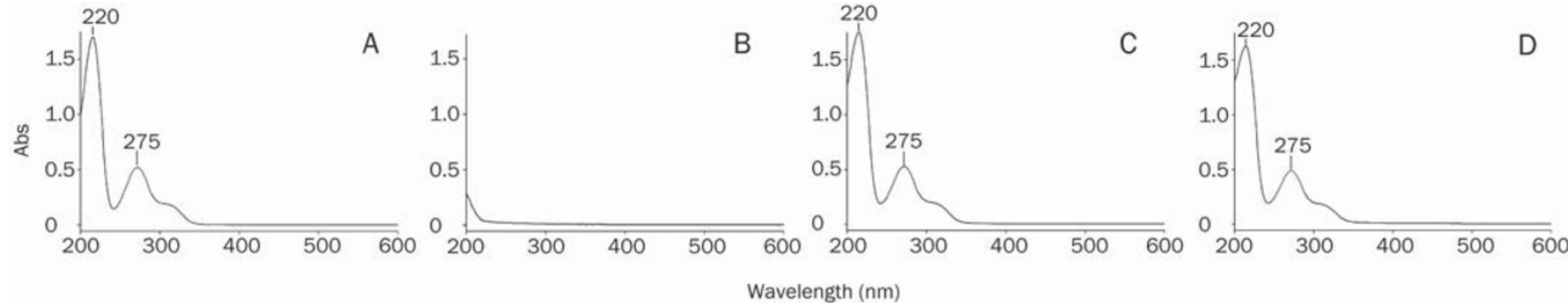

Figure 3. UV spectra of bergenin (A), phospholipids (B), physical mixture (C), and BPC (D).
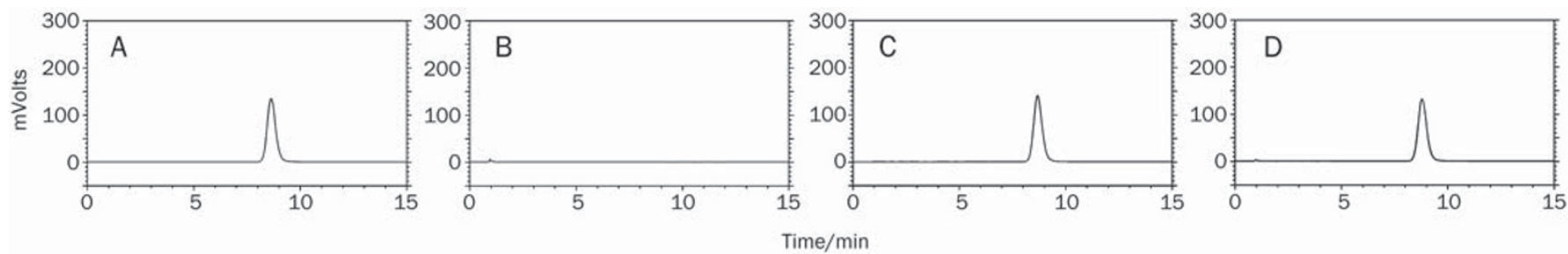

Figure 4. HPLC of bergenin (A), phospholipids (B), physical mixture (C), and BPC (D). The mobile phase was consisted of methanol-water (20:80, $v / v)$, $\mathrm{pH} 2.50$.
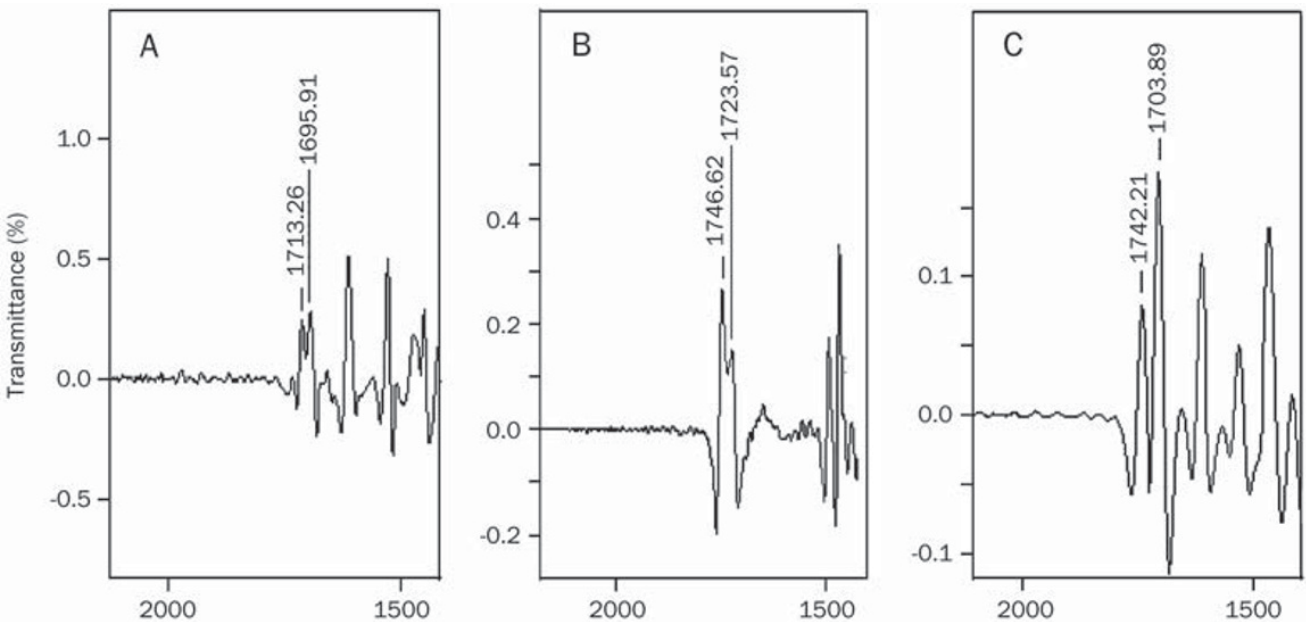

Figure 5. FT-IR spectra of bergenin (A), phospholipids (B), and BPC (C). 
the carbonyl absorptions $(\mathrm{C}=\mathrm{O})$ of bergenin and the phospholipids were at $1713 \mathrm{~cm}^{-1}$ and $1746 \mathrm{~cm}^{-1}$, respectively. Bergenin contains phenolic hydroxyl groups, and hydrogen bonding interactions between molecules was observed $\left(1695 \mathrm{~cm}^{-1}\right)$. The hydrogen bonds between molecules of phospholipids were present at $1723 \mathrm{~cm}^{-1}$. The hydrogen bonds in BPC $\left(1703 \mathrm{~cm}^{-1}\right)$ were different from the bonds in either bergenin or the phospholipids.

\section{Differential scanning calorimetry (DSC)}

DSC thermograms of bergenin (a), phospholipids (b), the physical mixture (c) and BPC (d) are shown in Figure 6. The thermogram of bergenin exhibited peaks at 143.0, 153.5, and $240.7^{\circ} \mathrm{C}$. The peak at $240.7^{\circ} \mathrm{C}$ was very sharp and appeared to be due to a phase transition from solid to liquid, which is in accordance with a report that $232-240{ }^{\circ} \mathrm{C}$ is the melting point of bergenin ${ }^{[17]}$. DSC of BPC showed that the endothermal peaks of bergenin and phospholipids disappeared.

\section{X-ray powder diffraction}

The powder of X-ray diffraction patterns of bergenin, the physical mixture and BPC are shown in Figure 7. Bergenin displayed partial sharp crystalline peaks, which is characteristic of a molecule with some crystallinity. The physical mixture exhibited both crystalline peaks and a wide peak due to the phospholipids. Compared with the above two, the crystalline peaks disappeared in the complex.

\section{Morphological characteristics of BPC}

The SEM and TEM photographs are shown in Figures 8 and 9. Bergenin exhibited a distinctive external crystal shape. BPC did not exist on the appearance of drug but appeared to be spherical with a smooth surface.

\section{Particle size and zeta-potential}

The particle size of BPC was distributed in a narrow range of $169.2 \pm 20.11 \mathrm{~nm}$, and the PDI was $0.212 \pm 0.032$. The zeta-potential is a key factor that could influence some properties of the particles, such as the stability in solution and interaction with other materials. However, no studies have been published to date in relation to this aspect of phospholipid complexes. The zeta-potential of BPC prepared in our experiment was $-21.6 \pm 2.4 \mathrm{mV}$, which might be related to the type and composition of the phospholipids.

\section{Solubility studies}

The data showed that the solubility of BPC in $n$-octanol or water was increased 177.32- and 6.03-times higher than bergenin, respectively (Table 4,5). The results also indicated that, for the physical mixture, the phospholipids enhanced the solubility of the drug, but this effect was weaker.

Table 4. Apparent solubility of bergenin and BPC in water at $25^{\circ} \mathrm{C} . n=3$.

\begin{tabular}{|c|c|c|c|c|}
\hline \multirow{2}{*}{ Sample } & \multicolumn{3}{|c|}{ Apparent solubility (mg/mL) } & \multirow{2}{*}{ Mean \pm SD } \\
\hline & 1 & 2 & 3 & \\
\hline Bergenin & 1.35 & 1.39 & 1.36 & $1.37 \pm 0.02$ \\
\hline Physical mixture & 1.55 & 1.46 & 1.36 & $1.46 \pm 0.08$ \\
\hline $\mathrm{BPC}$ & 10.38 & 8.96 & 9.55 & $9.63 \pm 0.58$ \\
\hline
\end{tabular}

Table 5. Apparent solubility of bergenin and BPC in $n$-octanol at $25^{\circ} \mathrm{C}$. $n=3$.

\begin{tabular}{|c|c|c|c|c|}
\hline \multirow{2}{*}{ Sample } & \multicolumn{3}{|c|}{ Apparent solubility (mg/mL) } & \multirow{2}{*}{ Mean $\pm S D$} \\
\hline & 1 & 2 & 3 & \\
\hline Bergenin & 0.71 & 0.70 & 0.74 & $0.72 \pm 0.02$ \\
\hline Physical mixture & 1.15 & 1.17 & 1.13 & $1.15 \pm 0.02$ \\
\hline $\mathrm{BPC}$ & 135.40 & 116.06 & 133.73 & $128.39 \pm 8.75$ \\
\hline
\end{tabular}

\section{Hygroscopicity studies}

In our experiments, we found that BPC could absorb moisture in the air and become viscous. Little information was available about this aspect, so the critical relative humidity $(\mathrm{CRH})$ was investigated. The weight increases of bergenin stored at $22.45 \%-98.00 \% \mathrm{RH}\left(25^{\circ} \mathrm{C}\right)$ are shown in Figure 10 . It was demonstrated that the bulk drug gained weight very slightly $(-0.03 \%-0.19 \% w / w)$, which suggested a good stability of bergenin in response to moisture during formulation and storage. When the drug was formed into the complex, the hygroscopicity was stronger. The $\mathrm{CRH}$ was about $80 \%$ at $25^{\circ} \mathrm{C}$, suggesting that BPC should be stored under relatively dry conditions $(\mathrm{RH}<80 \%)$.

\section{Pharmacokinetic study in rats}

After administration of a single dose $(300.0 \mathrm{mg} / \mathrm{kg})$ of ber-
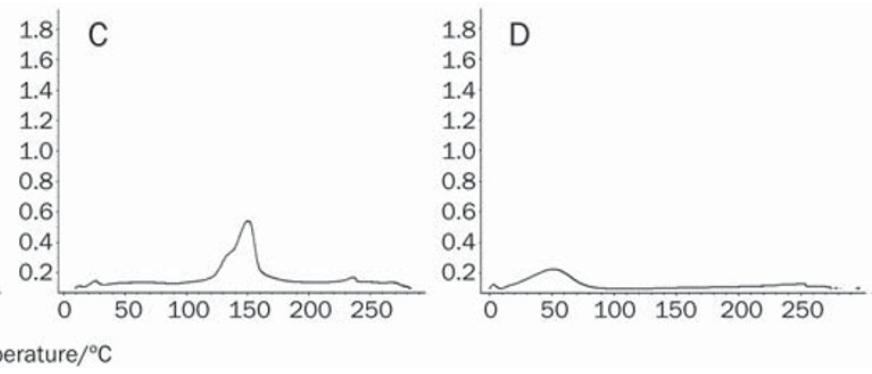
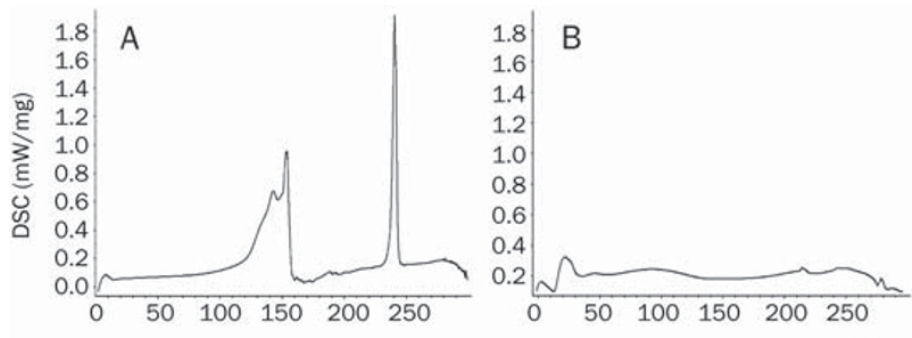

Figure 6. DSC thermograms of bergenin (A), phospholipids (B), physical mixture (C), and BPC (D). 

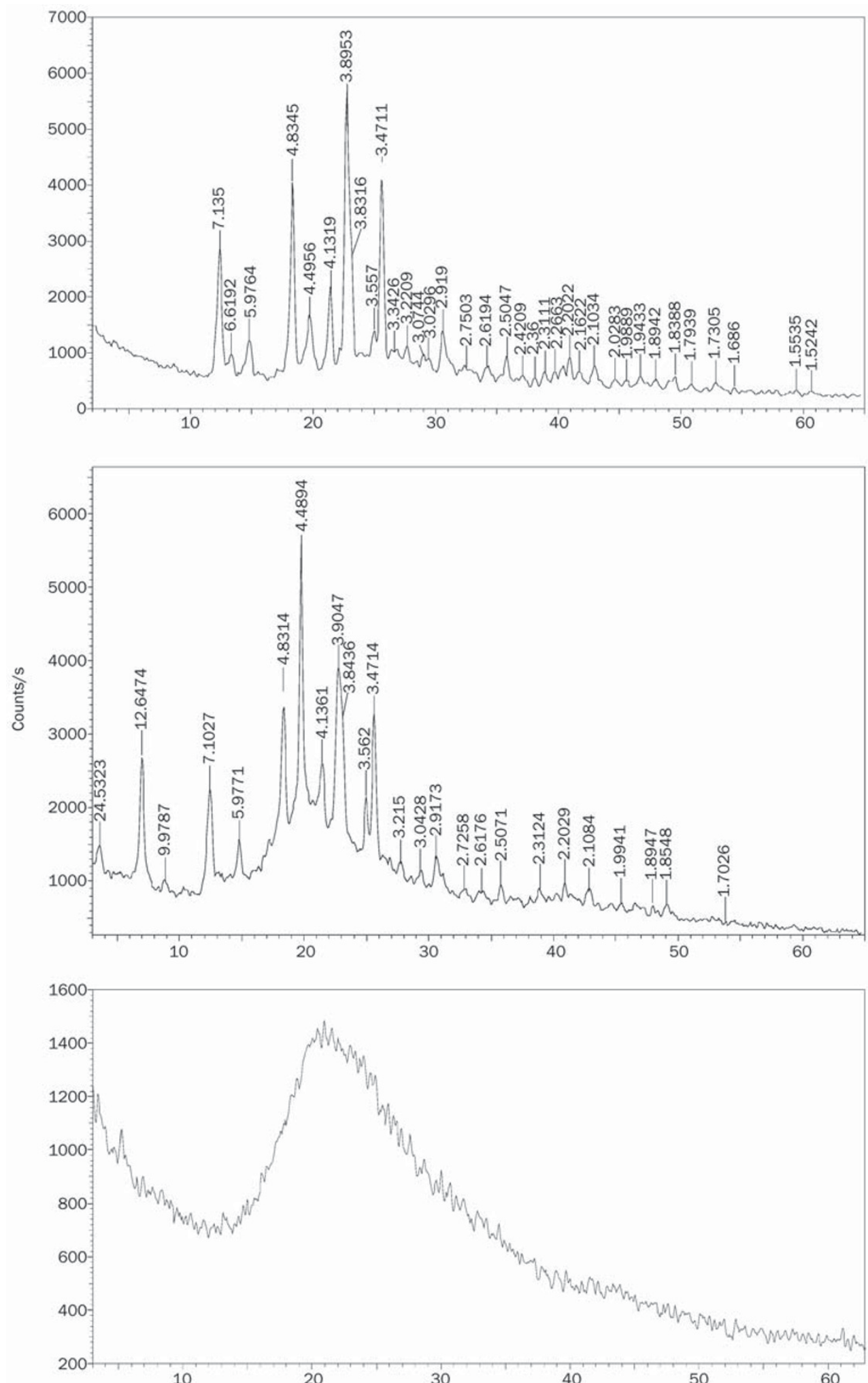

Figure 7. X-ray diffraction patterns of bergenin $(A)$, physical mixture $(B)$, and $\mathrm{BPC}(\mathrm{C})$.

genin, the physical mixture and BPC to rats, the plasma bergenin levels were analyzed over a $60 \mathrm{~h}$ period. The mean plasma concentration-time profiles are shown in Figure 11, and the pharmacokinetic parameters calculated from the data are summarized in Table 6 . The concentration-time pro- files were best described as a two-compartment open model and a biphasic phenomenon, with a rapid absorption followed by a slower elimination phase. As seen in the plasma curves, both the physical mixture and BPC showed higher plasma levels than free bergenin. For BPC, the $\mathrm{AUC}_{0 \rightarrow \infty}$ value 

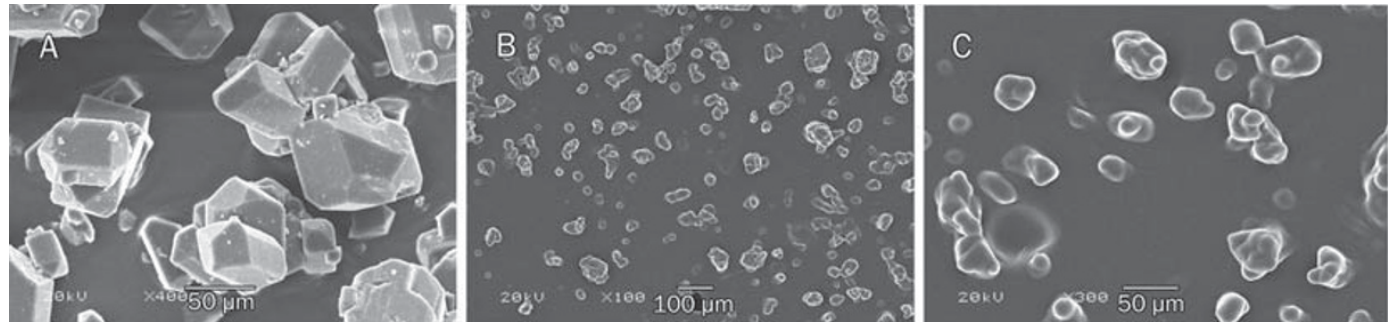

Figure 8. Scanning electron micrographs of bergenin at $\times 400$ (A) and BPC at $\times 100$ (B), and $\times 300$ (C) magnification.
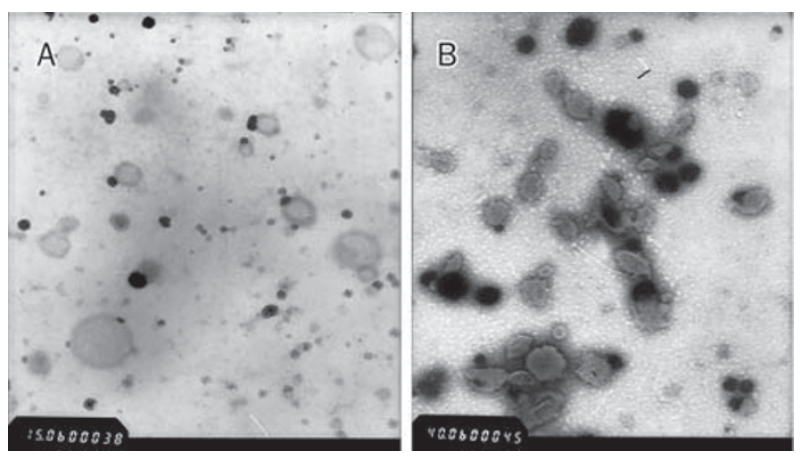

Figure 9. Transmission electron micrographs of BPC after slightly shaking in distilled water at $\times 1500$ magnification (A) and at $\times 4000$ magnification (B), respectively.

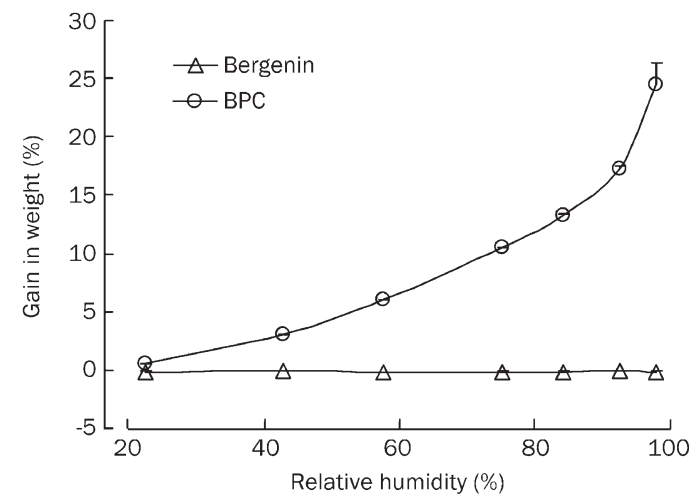

Figure 10. Moisture adsorption curve of bergenin and BPC.

Table 6. Main pharmacokinetic parameters of free bergenin $(300.0$ $\mathrm{mg} / \mathrm{kg}$ ), physical mixture and BPC (equivalent to $300.0 \mathrm{mg} / \mathrm{kg}$ of free bergenin) in rats after oral administration. $n=5$. Mean \pm SD.

\begin{tabular}{lccc}
\hline Parameter & Bergenin & Physical mixture & BPC \\
\hline$t_{1 / 2 \alpha}(\mathrm{h})$ & $9.16 \pm 3.66$ & $7.98 \pm 3.16$ & $1.12 \pm 0.16$ \\
$t_{1 / 2 \beta}(\mathrm{h})$ & $40.78 \pm 19.63$ & $20.74 \pm 10.84$ & $51.73 \pm 8.61$ \\
$\mathrm{MRT}_{0 \rightarrow \mathrm{t}}(\mathrm{h})$ & $21.31 \pm 1.70$ & $21.83 \pm 2.66$ & $30.30 \pm 4.95$ \\
$\mathrm{C}_{\max }(\mu \mathrm{g} / \mathrm{mL})$ & $0.44 \pm 0.06$ & $0.73 \pm 0.12$ & $2.87 \pm 1.29$ \\
$\mathrm{AUC}_{0 \rightarrow \infty}(\mu \mathrm{g} \cdot \mathrm{h} / \mathrm{mL})$ & $12.11 \pm 3.51$ & $18.91 \pm 8.20$ & $53.17 \pm 11.81$ \\
\hline
\end{tabular}

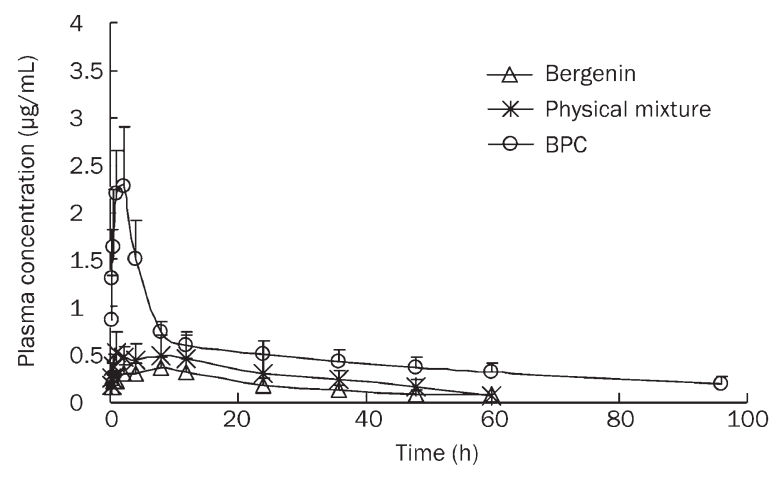

Figure 11. Plasma concentration profiles of bergenin in rat after oral administration of bergenin alone, physical mixture and BPC (equivalent to $300.0 \mathrm{mg} / \mathrm{kg}$ of bergenin). $n=5$. Mean \pm SD.

was $53.17 \pm 11.81 \mu \mathrm{g} \cdot \mathrm{h} / \mathrm{mL}$, and the relative bioavailability increased significantly to $439 \%$ of the control group. The $C_{\max }$ value $(2.87 \pm 1.29 \mu \mathrm{g} / \mathrm{mL})$ was almost 6.52 times that of bergenin $(0.44 \pm 0.06 \mu \mathrm{g} \cdot \mathrm{h} / \mathrm{mL})$. When the physical mixture was administered, a 1.56-fold higher $\mathrm{AUC}_{0 \rightarrow \infty}(18.91 \pm 8.20 \mu \mathrm{g} \cdot \mathrm{h} / \mathrm{mL})$ and 1.66-fold higher $C_{\max }(0.73 \pm 0.12 \mu \mathrm{g} / \mathrm{mL})$ were observed compared to the control group.

\section{Discussion}

In recent years, it has been reported that some poorly soluble drugs combined with phospholipids could result in an increase of oral bioavailability and/or improvement of the biological effects, such as silybin, curcumin and puerarin ${ }^{[18-20]}$. However, detailed information about the preparation of these complexes is limited. Peng et al used a central composite design approach for the optimization of ursodeoxycholic acid-phospholipid complex (UDCA). The yield (\%) of UDCA present as a complex was the unique evaluation index for the preparation $^{[21]}$. However, the content of drug in the complex that determines the pharmacologic action is another key factor and should not be neglected. In our experiment, both the combination percentage and content of the drug were taken into consideration. The spherical symmetric design-response surface methodology constitutes an alternative approach because it offers the possibility of investigating a high number of variables at different levels, while performing only a limited number of experiments ${ }^{[22]}$. In the experimental design of the 
spherical symmetric design-response surface methodology, the preliminary studies were investigated in detail. Organic solvents like chloroform, diethyl ether, acetone, methylenechloride, tetrahydrofuran, acetic ether, methanol and ethanol were investigated. A high combination percentage was achieved when methanol and ethanol were used. Considering that ethanol could be removed easily and is less harmful to the environment and human body compared with other solvents, it was used as the reaction medium. When the drug-tophospholipids ratio was lower than 0.4 , the resultant materials appeared viscous. When the ratio was more than 1.2 , crystals of bergenin could be observed in SEM photographs, and the combination percentage was low (these results were not shown).

In the literature, researchers have demonstrated that phospholipid complexes are not new chemical compounds or simple physical mixtures. Drugs and phospholipids should have some interactions, such as hydrogen bonding or van der Waals interactions, when they form complexes ${ }^{[18,23,24]}$. We performed some experiments to prove this point. In the structure of bergenin and the phospholipids, there were no chemical groups that could react with each other under our preparation conditions. Thus, it is hard for them to form a new substance. TLC was performed in order to determine whether a new substance was formed. In the chromatogram, no new spot was found. The result was further proven by UV and HPLC analyses. The chromatographic behavior of BPC was the same as bergenin, and no new peaks were found. This indicated that no new substance was formed in the preparation. However, the BPC showed different physical properties from bergenin and the physical mixture, such as increased solubility, different morphological characteristics and stronger hygroscopicity. Some interactions might exist between bergenin and phospholipids. In order to clarify the interactions, Fourier transform infrared spectral (FT-IR) analysis was performed. From the FT-IR spectra, the hydrogen bonds in BPC were different from the hydrogen bonds in either bergenin or the phospholipids. There are carbonyl groups belonging to phospholipids that allow the formation of hydrogen bonds with the phenolic hydroxyl groups of bergenin. This means that, when BPC was prepared, intermolecular coupling interactions changed and absorption bands attributed to the vibration of hydrogenbonded carbonyl groups were observed in the FT-IR spectra. With the hydrogen bonds between the molecules gone, the crystal shape of bergenin disappeared (from SEM photographs), and the sharp melting peak of bergenin was absent in DSC thermograms. The results of the X-ray diffraction studies also confirmed that in the phospholipids complex, bergenin was either molecularly dispersed or present as an amorphous form.

Phospholipids are an important component of cell membranes, having low toxicity and good biocompatibility. Due to their surface-active properties, phospholipids are commonly used as solubilizers or absorption enhancers to increase the permeability of some drugs. In the present study, the relative bioavailability of bergenin was significantly enhanced when it was administered as a phospholipid complex. In order to explore whether the enhanced absorption was the effect of phospholipids, a physical mixture of the drug and phospholipids was investigated and compared with the complex. The results showed that phospholipids could enhance both the $C_{\max }$ and relative bioavailability of bergenin, but the effect was weaker than in BPC $(P<0.05)$. It indicated that BPC could markedly improve the oral absorption of bergenin, and not just through the action of phospholipids. Small particle size, good solubility and permeability may be the main reasons for the improved absorption.

In conclusion, the preparation of BPC was optimized using the spherical symmetric design-response surface methodology by fitting a quadratic model to the response data. The experimental values of the BPC prepared under the optimum conditions were close to the predicted values and had a low percentage bias. The physicochemical properties and pharmacokinetic characteristics of BPC in rats were explored. Our results indicate that BPC has greatly enhanced solubility and improved oral bioavailability compared to bergenin and is more suitable for oral administration. However, the detailed mechanism of BPC oral absorption needs further investigation.

\section{Acknowledgements}

The research described above was supported by the National Basic Research Program of China (973 program, № 2009CB903301).

\section{Author contribution}

Xuan QIN, Yuan HUANG designed the research; Xuan QIN, Yang YANG, Ting-ting FAN and Tao GONG carried out laboratory experiments; and Xuan QIN, Yuan HUANG and Xiaoning ZHANG analyzed data and wrote the paper.

\section{References}

1 Nazir N, Koul S, Qurishi MA, Taneja SC, Ahmad SF, Bani S. Immunomodulatory effect of bergenin and norbergenin against adjuvantinduced arthritis-a flow cytometric study. J Ethnopharmacol 2007; 112: 401-5.

2 Ye YP, Sun HX, Pan YJ. Bergenin monohydrate from the rhizomae of Astilbe chinensis. Acta Crystallogr C 2004; 60: 397-8.

3 Wang G, Ma BJ. Overview of recent progress on bergenin. Anhui Zhong Yi Xue Yuan Xue Bao 2002; 21: 59-62.

4 Lim HK, Kim HS, Choi HS, Choi J, Kim SH, Chang MJ. Effects of bergenin, the major constituent of Mallotus japonicus against $D$-galactosamine-induced hepatotoxicity in rats. Pharmacology 2001; 63: 71-5.

5 Piacente S, Pizza C, Tommasi N, Mahmood N. Constituents of Ardisia japonica and their in vitro anti-HIV activity. J Nat Prod 1996; 59: 565-9.

6 Takahashi H, Kosaka M, Watanabe Y, Nakade K, Fukuyama Y. Synthesis and neuroprotective activity of bergenin derivatives with antioxidant activity. Bioorg Med Chem 2003; 11: 1781-8.

7 Jiangsu New Medical College. Traditional Medicine. Shanghai, China: Shanghai People's Publishing House 1999; p 2359.

8 Lipkaa E, Amidonb GL. Setting bioequivalence requirements for drug development based on preclinical data: optimizing oral drug delivery 
systems. J Control Rel 1999; 62: 41-9.

9 Dan Z, Xuan Q, Zhi RZ, Yuan H. Physicochemical properties of bergenin. Pharmazie 2008; 63: 366-71.

10 Cyril R, Olivier RM. Synthesis of bergenin-related natural products by way of an intramolecular C-glycosylation reaction. TetrahedronAsymmetr 2000; 11: 409-12.

11 Wang G, Liu JK, Ma BJ. The acetylate of bergenin and its application. PR China Patent 2006; 200510010970.0.

12 Li XD, Lu SL, He B. The preparation of bergenin compositae pills. PR China Patent 2005; 200410040660.9.

$13 \mathrm{Li} \mathrm{J}$. The preparation of bergenin compositae soft capsules. PR China Patent 2005; 200410078846.3.

14 Guan JY, Sun DJ, Wu GL. The study of bergenin's inclusion complex. Zhong Cheng Yao 1991; 13: 4-5.

15 Stokes RH. Standard solutions for humidity control at $25^{\circ} \mathrm{C}$. Ind Eng Chem 1949; 41: 2013.

16 Xuan Q, Dan Z, Zhi RZ, Yuan H. Determination of bergenin in rat plasma by high-performance liquid chromatography. Pharmazie 2007; 62: 323-6.

17 Chinese Pharmacopoeia Commission. The Pharmacopoeia of the People's Republic of China 2005; part I: 278.

18 Xiao YY, Song YM, Chen ZP, Ping QN. The preparation of silybin- phospholipid complex and the study on its pharmacokinetics in rats. Int J Pharm 2006; 307: 77-82.

19 Kuntal M, Kakali M, Arunava G, Bishnu, PS, Pulok KM. Curcuminphospholipid complex: Preparation, therapeutic evaluation and pharmacokinetic study in rats. Int J Pharm 2007; 330: 155-63.

20 Li Y, Pan WS, Chen SL, Xu HX, Yang DJ, Chan ASC. Pharmacokinetic, tissue distribution, and excretion of puerarin and puerarin-phospholipid complex in rats. Drug Dev Ind Pharm 2006; 32: 413-22.

21 Peng FY, Zhang WJ, Yuan HL, Yang M, Zhu WF, Cai PL, et al. Process optimization, characterization and pharmacokinetic evaluation in rats of ursodeoxycholic acid-phospholipid complex. AAPS PharmSciTech 2008; 9: 322-9.

22 Wu W, Cui GH. Central composite design-response surface methodology and its application in pharmaceutics. Foreign Med Sci Sect Pharm 2000; 27: 292-8.

23 Venema FR, Weringa WD. The interactions of phospholipid vesicles with some anti-inflammatory agents. J Colloid Interface Sci 1988; 125: 484-500.

24 Peng FY, Hai LY, Huan X, Xiao HX, Ming Y, et al. Preparation, characterization, and bioavailability of ursodeoxycholic acid-phospholipid complex in vivo. Drug Dev Ind Pharm 2008; 34: 708-18. 